\title{
Stimulating science
}

\author{
The economic benefits of biomedical research are recognized by governments around the world, but investment \\ in science should go beyond profitability.
}

W ith the world entering recession, governments around the globe are investing in science as part of a larger stimulus package. In the United States, the House and Senate are hammering out the differences between their versions of an economic stimulus bill that will channel billions of dollars of additional funding into biomedical research and a smaller amount into physical and earth sciences.

This welcome news recognizes the economic benefits of scientific research but places scientists and funding agencies under more pressure. The importance of the economic stimulus is underlined by the level of scrutiny attached, with oversight committees tasked with rooting out inefficiency and the launch of the website Recovery.gov (http://www.recovery.gov/) for citizens to ensure that no tax dollars are wasted. And so the major research funders will need to allocate their grants carefully-

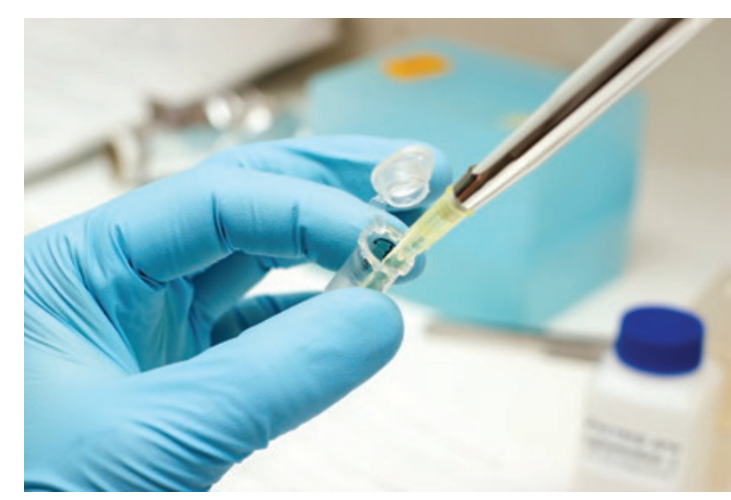

scientific standing and to close the gap with the United States. India and China, for example, have already recognized that their futures depend on their investment in science, and Canada has included money for science infrastructure within its own stimulus budget. The United Kingdom has pledged to maintain its science budget, despite the economic downturn. All agree that research has economic benefits.

A recent report from the UK Medical Research Council, Wellcome Trust and Academy of Medical Sciences backs this view, calculating that biomedical research has an annual rate of return for investment of around 39\% per year, with immediate benefits to the local community through jobs and purchasing of equipment and reagents, and long-term benefits for health and quality of life.

But while recognizing the importance of science in its present form to the economy, the UK science minister and quickly, too, if the House proposal that the US National Science Foundation (NSF) and Department of Education must spend their funds within 120 days remains in the final version.

Much of the money is earmarked for research grants to individuals and small groups, and the principal winners of this funding, the US National Institutes of Health (NIH) and NSF, will need to spend it in a way that avoids funding problems 2 years down the line when the stimulus ends. Without knowing whether this is a one-off boost or part of a long-term increase in science funding, the agencies are likely to take a cautious approach.

The NIH and NSF have no shortage of attractive projects that they could fund, with the acting director of the NIH, Raynard S. Kington, estimating that it could quickly fund the 14,000 applications over the past 6 years that it was forced to turn down because of insufficient financing, despite their academic merit. The NSF expects grants to go to the applications already submitted from 1 October 2008 and fund those most highly rated by reviewers. The money could go towards funding younger investigators and riskier projects, which is all the more important considering that the number of tenure-track and tenured positions in the United States has not increased in the past 2 decades.

The increase in funding for science in the United States is likely to have a knock-on effect to other governments striving to improve their
Lord Drayson has called for a debate on whether this money should be spent on areas of science that are compatible with industrial and economic priorities. This would be a fundamental change in how science is funded, moving away from choosing projects on their academic merit-the so-called Haldane Principle-to choosing on their economic usefulness.

The United States leads the way in commercially exploiting science, and the United Kingdom, despite pioneering work in many fields, has often failed to turn the advantage into profit. The UK Treasury is now beginning to ask what the public is getting for the doubling of the science budget over the past 10 years.

But medical advances do not happen without investment in fundamental scientific research. Science is serendipitous, and it is almost impossible to predict where the breakthroughs and commercially successful scientific innovation will come from.

The fact that so many countries recognize the economic benefits of scientific research is encouraging, but we must resist the pressure to constrain our research to that which looks likely to make money. We must not let the economic case blind us to the importance of maintaining a wide, multidisciplinary and long-term research base. Without investment in basic research, there will be nothing to translate into profit. 